\title{
A brave new virtual conferencing world
}

\author{
Travel restrictions due to COVID-19 have forced the science world to change conference and networking practices. \\ Retaining the positives of virtual interactions should be a priority in the post-pandemic era.
}

T he travel restrictions and strict health and safety guidelines put in place since the beginning of the year due to the COVID-19 pandemic resulted in a seismic shift in the way scientists interact. As universities, research institutes and companies put non-essential work-related travel on hold, the scientific conferences set to take place in 2020 were canceled, postponed or moved to virtual formats, with plans for 2021 meetings being evaluated as the global COVID-19 situation continues to develop.

Indeed, rather than scrapping planned meetings altogether, several organizations rapidly switched to digital platforms no mean feat, given that many had limited, if any, experience on that front. Thus, some of the mainstays of the yearly cancer-conference circuit, such as the annual meetings of the American Association for Cancer Research (AACR) and the American Association of Clinical Oncology (ASCO), took the form of virtual mega-conferences, with the European Society for Medical Oncology also planning a virtual congress in the fall. The transition to digital conferencing was embraced enthusiastically by the community, with registration for the ASCO annual meeting matching and for the AACR meeting largely exceeding their typical numbers of many tens of thousands of in-person attendees. Smaller, more focused conferences planned by these and other organizations also made the successful move to an online environment.

There are many advantages to going virtual. The ability to maintain academic interactions digitally has helped provide a level of continuity in science exchange, even as many labs had to close temporarily due to the pandemic. Beyond enabling the participation of those scientists who had to cancel specific travel plans, the move to digital formats has democratized attendance at conferences by making them more accessible to a wider and more diverse group of researchers worldwide. The reduced costs associated with virtual participation permit the registration of scientists who have limited financial means or live far from areas that are traditional conference hubs. Removing the need to travel opens participation to those for whom traveling might be more complicated or unfeasible due to visa requirements, pressing family obligations and caregiving responsibilities, or disabilities. Apart from eliminating the geographical restrictions of physical attendance, the move to online conferences reduced the carbon footprint of individual researchers who are frequent travelers, and of the scientific conference enterprise as a whole. As an example, the carbon emissions of the Fall Meeting of the American Geophysical Union, an international conference that was held in San Francisco last year with attendance similar to that of the AACR Annual Meeting, was calculated to be on a par with the average weekly emissions of the city of Edinburgh, UK. In addition, the flexibility afforded by attending a conference from the comfort of one's own home may permit more focused and productive participation, especially for larger meetings that might feel more chaotic in person. Virtual conferences could also offer the possibility of broader audience participation in question-and-answer sessions - for example, by emboldening junior participants who might feel less at ease with asking questions in an auditorium, or by permitting the audience to vote on questions in real time, thus elevating the quality of the scientific discourse.

Given the benefits of online meetings, is 2020 going to be the year when scientists stop traveling to conferences? Health factors aside, such predictions may be quite premature. Although going digital has in principle permitted conference attendance by a global audience, in practice, time-zone considerations remain limiting for speakers and attendees, making many online conferences more regional affairs than their potential for a global reach might imply. The reliance on technology and connectivity brings the possibility of technical disruptions, something that organizers, speakers and attendees have been largely taking in stride this year, especially given that many meetings became virtual at short notice. Nevertheless, however smoothly an online meeting may run, a hard-to-capture, missing ingredient is the vibe set by real-life interactions. The lack of a physical audience and the need to sometimes pre-record presentations deprive speakers of the ability to 'read the room' and tailor their talk to the listeners' reactions or engage with them more directly. It also deprives the audience from getting to know something of the speaker's personality and seeing the person behind the science. Indeed, the interactive aspect of conferences is sometimes almost entirely lost, especially when audience participation is limited to submitting questions after a talk. Poster sessions, that very important and very social component of scientific conferences, rely on participants engaging in many short conversations during a short period of time and may not translate successfully to the virtual setting. This might discourage some presenters, especially more junior ones, for whom presenting and networking are crucial for career development. Moreover, the fortuitous discussions and connections that so often happen at conferences due to the random seating arrangements, or during breaks and mealtimes, and may lead to new research directions, collaborations and friendships are absent from the online space. Finally, after half a year of relying on technology for much of our human interaction, be it through video chats with family, friends and colleagues, or online work-related meetings, video-meeting fatigue has set in for many researchers, who may feel that filling their schedules with yet more screen time to attend an online conference might not be very beneficial.

Humans are social beings and thrive with direct interactions, so in-person meetings are bound to start again when it is safe to hold them. However, that does not mean we should discount the success and wide accessibility of digital conferencing. With the pandemic still unabated, it is hard to envision an exclusive return to physical conferences, with hundreds or thousands of people traveling to far-flung locations to spend days together in closed auditoria, milling around poster sessions and sharing food and drink while discussing science. Perhaps, then, 2020 will be the year when the unwelcome push of COVID-19 spurred scientists to rethink how they run scientific conferences and to seek more inclusive and sustainable modes of scientific exchange for the future. That could involve providing a mix of conferences with online and physical attendance, adding virtual components to in-person events, and incorporating more interactive virtual networking sessions, aiming to foster participatory diversity and support trainees and junior investigators. 
Online conferences cannot emulate the atmosphere and intimacy of attending a scientific conference in person, but that is $\mathrm{OK}$. Just as meeting someone in real life is different from a video chat, attending a virtual conference serves different needs. It is up to the scientific community to ensure that we reap the benefits of both the physical and virtual conferencing worlds by making the most of the available technologies for fruitful scientific interactions.

The Nature Cancer team is always available for virtual interactions with our authors and readers, so do not hesitate to reach out to us at cancer@nature.com or by getting in touch with specific editors.

Published online: 18 August 2020

https://doi.org/10.1038/s43018-020-00108-1 